\title{
Successful use of caudal anesthesia and light sevoflurane mask ventilation for inguinal hernia repair in an infant with multiple large intrapulmonary cysts
}

\author{
Jae-Wook Jung, Seong Rok Kim, Sang Yoon Jeon, Yong Han Kim, and Si Ra Bang \\ Department of Anesthesiology and Pain Medicine, Haeundae Paik Hospital, Inje University College of Medicine, Busan, Korea
}

Cystic lung diseases of infants include congenital cystic adenoid malformation, pulmonary sequestration, bronchogenic cyst, and bronchial atresia. The progressions of cystic lung disease vary from spontaneous regression to severe respiratory difficulty [1]. Infants with cystic lung disease can suffer variable degrees of respiratory symptoms which can result in recurrent infection and pulmonary hypoplasia [2]. Therefore, perioperative management of patients with cystic lung disease can be a challenge for anesthesiologists. We report our experience with a 2-month-old female patient with multiple large intrapulmonary cysts.

The 2-month-old-female patient was scheduled for surgery for a right-side inguinal hernia. The baby was born prematurely at 36 weeks, weighing $3.4 \mathrm{~kg}$. She was admitted to the neonatal intensive care unit for 4 days right after birth. She was asymptomatic at birth and doing well after discharge. The patient was diagnosed with inguinal hernia and scheduled for surgery.

In the preoperative evaluation, chest $\mathrm{X}$-rays showed a unilocular air-containing cystic lesion of $4.8 \times 3.3 \mathrm{~cm}$ in size occupying the left upper lung region (Fig. 1A). She was checked via a computed tomography (CT) scan. The CT scan revealed a large air-containing cyst in left upper lung and several small cysts in right upper lobe, right middle lobe, and both lower lobes (Fig. 1B).

In the operating room, she was monitored through pulse oximetry, non-invasive arterial pressure and electrocardiographic monitoring. She received $1 \mathrm{mg} / \mathrm{kg}$ ketamine intravenously. The patient was placed in the right lateral position. After local anesthesia with $1 \%$ lidocaine, we used $0.25 \%$ bupivacaine $1 \mathrm{ml} / \mathrm{kg}$ for the caudal block with 22-gauge angiocatheter using an ultrasound machine (Philips CX50, Philips ultrasound, Bothell, WA, USA). She turned into the supine position right after the injection. After 10 minutes, we confirmed motor block of the lower extremities. There was no lower extremity movement during the operation. Blood pressure and heart rate were maintained within baseline values. During the operation, we used spontaneous breathing light sevoflurane for sedation via a face mask.

The total operation time was 20 minutes. She was transferred to the postanesthetic care unit with intact self-respiration. She was discharged the next day uneventfully.

A caudal block is a commonly performed technique during anesthesia of pediatric patients. It is known to be used as the sole form of anesthesia instead of as general anesthesia for highrisk neonate patients. Our institution usually performs general anesthesia in infants for inguinal hernia repair. However, in this case, we considered the use of regional anesthesia to avoid unintentional complications.

A variety of congenital cystic lung lesions may appear in the neonatal period. The development of prenatal care has made frequent the detection of congenital cystic lung disease via fetal ultrasonography [1]. The cystic lesion in her CT scan represented large air-containing cyst in the left upper lung and several small

Corresponding author: Si Ra Bang, M.D., Ph.D., Department of Anesthesiology and Pain Medicine, Haeundae Paik Hospital, Inje University College of Medicine, 875, Haeun-daero, Haeundae-gu, Busan 612-896, Korea. Tel: 82-51-797-0421, Fax: 82-51-797-0499, E-mail: sira1045@naver. com

(C) This is an open-access article distributed under the terms of the Creative Commons Attribution Non-Commercial License (http:// creativecommons.org/licenses/by-nc/3.0/), which permits unrestricted non-commercial use, distribution, and reproduction in any medium, provided the original work is properly cited. 

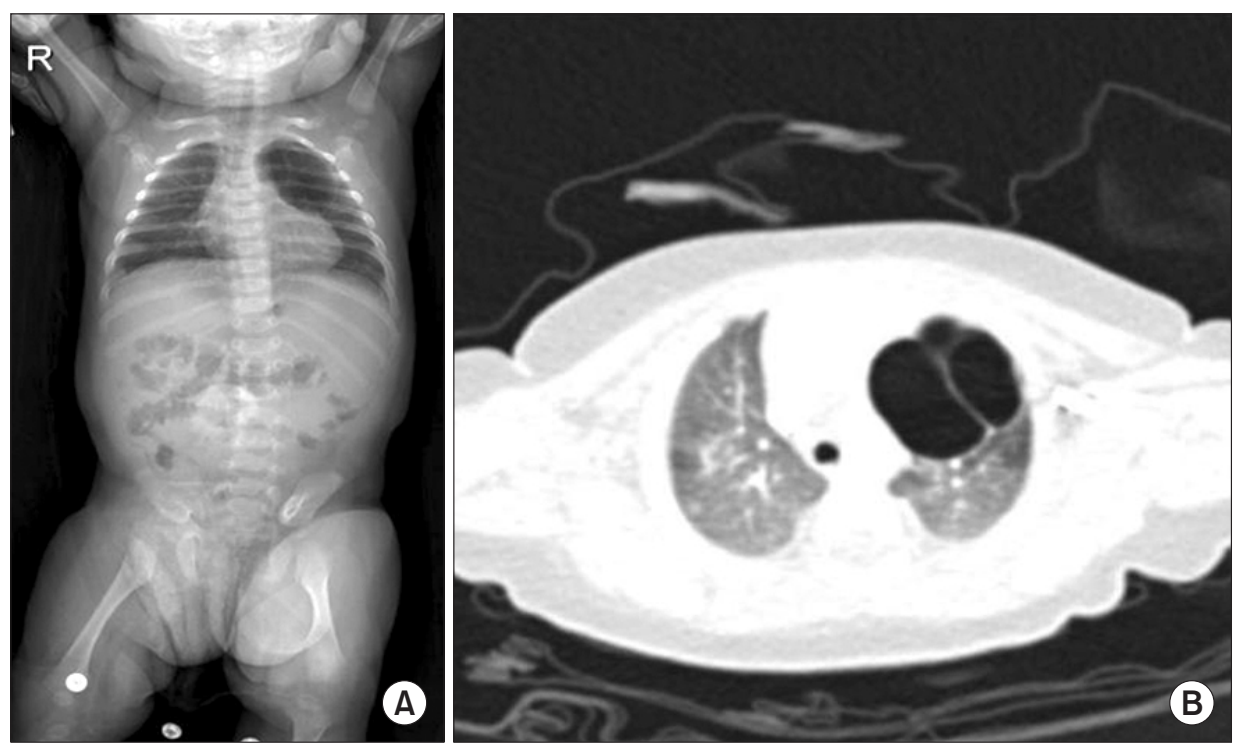

Fig. 1. Preoperative infantogram. An unilocular air-containg cystic lesion $(4.8$ $\times 3.3 \mathrm{~cm}$ ) in left upper lung (A). CT scan represented large air-containing cyst in the left upper lung and several small cysts in multiple lobes (B).

cysts in multiple lobes. There are few reports of the anesthetic management of cases of inguinal hernia repair in infants with multiple intrapulmonary cysts.

Our anesthetic concern was to prevent barotraumas due to positive pressure ventilation. Large cystic lesions can induce tension pneumothorax and can further cause cardiovascular collapse. Therefore we decided to utilize regional anesthesia with spontaneous mask ventilation. Another concern was the risk of postoperative apnea. Before reaching the postconceptional age of 60 weeks, ex-premature infants are at risk of postoperative apnea [3]. Our patient's postconceptional age was 44 weeks so the risk of apnea still remained. There are several anesthtetic techniques to avoid postoperative apnea including caudal or spinal anesthesia. Caudal anesthesia has considerably easier technical requirements and for that reason is performed widely. It is known to a safe and effective procedure for pediatric patients undergoing subumbilical surgery. However, there remain some problems such as the presence of pain and the possibility of an inadequate anesthetic level.

Geze et al. [4] reported a successful case of caudal anesthesia during inguinal hernia repair of a low-birth-weight neonate while awake. However, we used ketamine during caudal block for relief in patients with stressful conditions. During the operation, we established light sevoflurane mask ventilation and maintained self-respiration without any adverse events. The epidural space of infants is different from that of adults due to increased vascularity, less fat, and a smaller absorptive surface for local anesthetics [5]. So, we used ultrasonography to ensure safety and accuracy of the regional block. It provided the advantage of detecting sacral anatomy and of visualing the spread of local anesthetics. Therefore, the side effects of a caudal block, such as an inadvertent vascular or dural injection, can be minimized. The combination of sevoflurane and caudal anesthesia may prevent movement and pain during surgery in accordance with a stable hemodynamic condition.

In conclusion, the anesthesic care of infants with cystic lesions can be challenging for anesthesiologists. We report a successful single-shot caudal block under light sevoflurane sedation. It can be used effectively for performing a procedure on a high-risk infant.

\section{References}

1. Usui N, Kamata S, Sawai T, Kamiyama M, Okuyama H, Kubota A, et al. Outcome predictors for infants with cystic lung disease. J Pediatr Surg 2004; 39: 603-6.

2. Chow PC, Lee SL, Tang MH, Chan KL, Lee CP, Lam BC, et al. Management and outcome of antenatally diagnosed congenital cystic adenomatoid malformation of the lung. Hong Kong Med J 2007; 13: 31-9.

3. Welborn LG. Postoperative apnoea in the former preterm infant: a reviw. Paed Anaesth 1992; 2: 37-44.

4. Geze S, Imamoğlu M, Cekic B. Awake caudal anesthesia for inguinal hernia operations: successful use in low birth weight neonates. Anaesthesist 2011; 60: 841-4.

5. Suresh S, Polaner DM, Cote CJ. Regional anesthesia. In: A Practice of Anesthesia for Infants and Children. 5th ed. Edited by Cote CJ, Lerman J, Anderson BJ: Philadelphia, Elsevier Saunders. 2013, p 836. 